\title{
Validation of the V-index as a metric of ventricular repolarization dispersion using intracardiac recordings
}

\author{
M Orini ${ }^{1}$, C Blasi ${ }^{2}$, M Finlay ${ }^{3}$, B Hanson ${ }^{4}$, P Lambiase $^{1}$, R Sassi $^{5}$, LT Mainardi $^{2}$ \\ ${ }^{1}$ Institute of Cardiovascular Science, University College London, United Kingdom \\ ${ }^{2}$ Dipartimento di Elettronica, Informazione e Bioingegneria, Politecnico di Milano, Italy \\ ${ }^{3}$ William Harvey Research Institute, Queen Mary University of London, United Kingdom \\ ${ }^{4}$ Department of Mechanical Engineering, University College London, United Kingdom \\ ${ }^{5}$ Dipartimento di Informatica, Universitá degli Studi di Milano, Italy
}

\begin{abstract}
The spatial heterogeneity of ventricular repoalarization $($ SHVR) is an important factor in arrhythmogenesis and it is related to increased vulnerability to fatal arrhythmia. The V-index is a non-invasive estimator of SHVR based on the analysis of the surface ECG. A significant correlation between the V-index and SHVR has been previously demonstrated theoretically and by means of numerical simulations. Also, the V-index has been shown to track expected changes in SHVR after infusion of drugs that alter the cardiac electrophysiology. In this study, we compare for the first time estimates from the V-index with direct measures of SHVR derived from unipolar elecrograms simultaneously recorded in the left and right ventricular endocardium and in the left ventricular epicardium. In 22 recordings collected in 14 patients with normal ventricles, the $V$-index showed a tight and significant correlation with directed measures of SHVR, with correlation coefficients as high as $r=0.80$, whereas other ECG-derived estimates of SHVR based on T-peak and T-peak to T-end measures showed lower correlations ( $r \leq 0.41$ and $r \leq 0.36$, respectively). The results of this study suggest that the $V$ index provides reliable estimates of SHVR.
\end{abstract}

\section{Introduction}

The coordination of the electrical excitation and repolarization throughout the ventricular myocardium allows the heart to contract and pump blood. An alteration of electrical conduction and repolarization can result in a deterioration of the cardiac function or trigger dangerous arrhythmia. The spatial heterogeneity of ventricular repolarization (SHVR) refers to a nonhomogeneous recovery of excitability and it is an important factor in arrhythmogenesis [1]. An increased dispersion of repolarization constitutes an arryhthmogenic substrate, that in presence of a trigger (such as an ectopic beat) can lead to functional conduction block, re-entry, ventricular tachycardia/fibrillation and eventually sudden cardiac death [2]. Therefore, to prevent sudden cardiac death and better understand mechanisms of arrhythmia, a methodology that provides an accurate quantification of SHVR from non-invasive measurements is needed. The surface ECG provides non-invasive and cheap measurements of the electrical activity of the heart and can be used to study cardiac repolarization $[3,4]$. In particular, the morphology of the T-wave is associated with ventricular repolarization [5] and parameters that quantify its morphology have been shown to have a predictive value [6].

Recently, the V-index has been proposed as an ECGderived marker of SHVR [7], and has already shown promising results [8-10]. With respect to the majority of other non-invasive indices of SHVR, the V-index presents two advantages. Firstly, being based on a biophysical model [11] it provides a clear interpretation of what it is measuring. Secondly, the methodology is robust against noise and artifacts because it analyzes the morphology of the T-wave instead of relying on the localization of few fiducial points (as for instance the T-peak or T-end).

The aim of this study is to provide a validation of the Vindex as a marker of SHVR, by comparing it with measures of dispersion obtained simultaneously from unipolar electrograms (UEG) recorded in the left and right ventricular endocardium as well as in the left ventricular epicardium.

\section{Experimental protocol}

Studies were performed in 14 patients undergoing cardiac electro-physiological studies with a view to diagnosis and ablation of supraventricular tachycardia as described in [12]. All patients gave prior informed consent. Studies 
were performed under minimal conscious sedation in the post-absorptive state. Antidysrhythmic drugs were discontinued for 5 days prior to the study. The study protocol had local ethics committee approval and conformed to the standards set by the Declaration of Helsinki. ECG was recorded in the standard 12-lead configuration. UEG were recorded as follows. Decapolar catheters were placed in an epicardial coronary vein via the coronary sinus, at the right ventricular apex and retrogradely within the left ventricular cavity adjacent to the epicardial catheter (St Jude Pathfinder). A reference anodal electrode was placed in the inferior vena cava. UEG and ECG were sampled at 2 $\mathrm{KHz}$ (Bard Clearsign, MN, USA). Ventricular pacing was delivered from the right ventricular apex during baseline conditions, mental stress and after infusion of Ajmaline, a drug that slows electrical conduction and prolongs action potential duration. Only recordings including more than 45 beats, and presenting high signal to noise ratio (SNR $>15$ $\mathrm{dB}$ ) and few abnormal beats were considered. After selection, 22 recordings ( 9 baseline, 9 stress, 4 Adjmaline) were included in the study. Example of recordings are shown in Fig. 1.

\section{Methods}

\subsection{V-index calculation}

The V-index is derived from a simplified version of the bidomain model of the myocardium [13], in which the potential recorded at the body surface during a cardial cycle $k$ can be written as [7,9]:

$$
\boldsymbol{\Psi}(t)=\mathbf{A}\left[\begin{array}{c}
D_{1}(t) \\
\cdots \\
D_{M}(t)
\end{array}\right] \approx \underbrace{\mathbf{A} \boldsymbol{\Delta} \rho}_{\mathbf{w}_{\mathbf{1}}} T_{\mathrm{d}}(t)+\underbrace{0.5 \mathbf{A} \boldsymbol{\Delta} \rho^{2}}_{\mathbf{w}_{\mathbf{2}}} \dot{T}_{\mathrm{d}}(t)
$$

In this expression, $\mathbf{A}=\left[A_{\mathrm{i}, \mathrm{m}}\right]$ is the transfer matrix from the transmembrane potentials $D_{\mathrm{m}}(t)$ evaluated at intracrdiac sources $m=1, \ldots, M$ and the potentials $\Psi_{\mathrm{i}}(t)$ recorded on the body surface in correspondence of cardiac leads $i=1, \ldots, L$. The model assumes that the repolarization phase of each transmembrane potential can be written as $D_{\mathrm{m}}(t)=D\left(t-\rho_{\mathrm{m}}\right)$, i.e. it is obtained by translating a constant $D(t)$ by the local repolarization time $\rho_{\mathrm{m}}(k)=\bar{\rho}(k)+\Delta \rho_{\mathrm{m}}(k)$, where $\bar{\rho}(k)$ is the mean repolarization time calculated across the $M$ sources. In (1), $T_{\mathrm{d}}(t)=-\dot{D}_{\mathrm{m}}\left(t-\bar{\rho}_{\mathrm{m}}\right)$. In [7], the spatio-temporal distribution of repolarization delays $\Delta \rho_{\mathrm{m}}(k)$ across cardiac sites $m$ and heart beats $k$ was modeled as:

$$
\Delta \rho_{\mathrm{m}}(k)=\vartheta_{\mathrm{m}}+\varphi_{\mathrm{m}}(k)
$$

where $\vartheta_{\mathrm{m}}$ and $\varphi_{\mathrm{m}}(k)$ represent spatial and temporal variability, respectively. The V-index is an estimate of the standard deviation of $\vartheta_{\mathrm{m}}, s_{\vartheta}$, which according to the model is

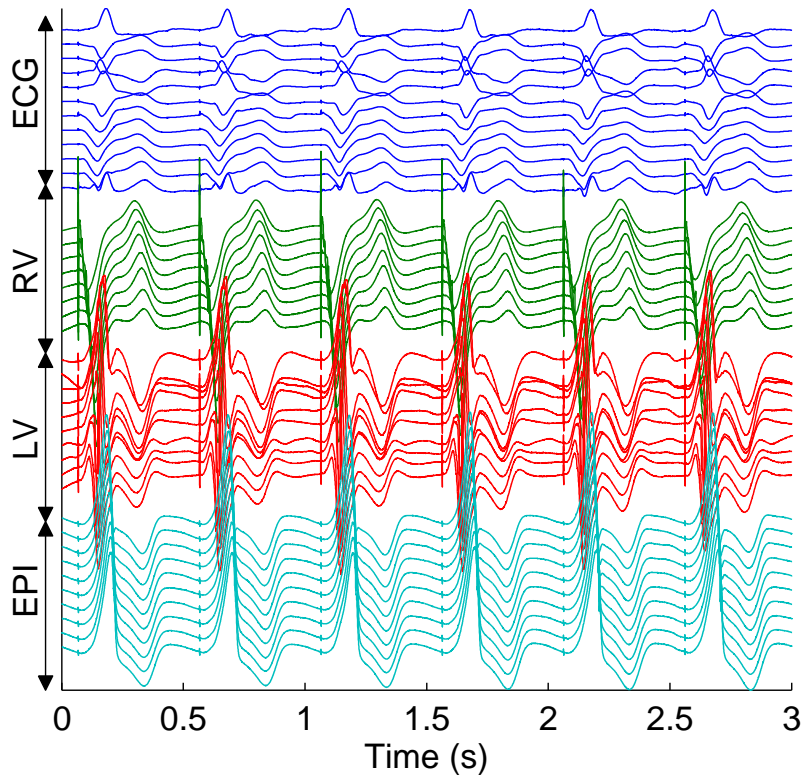

Figure 1. Illustrative example of data (high pass filtered to remove baseline trends), including 12-leads ECG and unipolar electrograms from the right (RV) and left (LV) ventricular endocardium, as well as from left ventricular epicardium (EPI). Spikes represent RV pacing, delivered at $t_{\mathrm{i}}^{0}(k)$.

a measure of SHVR. In particular, as shown in [7], the Vindex is obtained as:

$$
\mathcal{V}_{\mathrm{i}}=\frac{\operatorname{std}_{\mathrm{k}}\left[w_{2, \mathrm{i}}(k)\right]}{\operatorname{std}_{\mathrm{k}}\left[w_{1, \mathrm{i}}(k)\right]} \approx s_{\vartheta}
$$

In this study, the standard deviations calculated across heart beats $k$ were estimated in a robust way by using the median of absolute differences, defined as $\operatorname{median}_{\mathrm{k}}[\mathrm{X}(\mathrm{k})-\operatorname{median}[\mathrm{X}(\mathrm{k})]]$. The average of $\mathcal{V}_{\mathrm{i}}$ across leads $i$ was taken as final measure of SHVR.

\subsection{Repolarization analysis}

The analysis of UEG and ECG was carried out by means of bespoke algorithms as in previous studies [14-16]. As shown in the diagrammatic example of Fig. 2, local repolarization times were measured as the maximum of the first derivative of the $i$-th UEG channel, i.e. $t_{\mathrm{i}}^{\mathrm{R}}(k)=$ $\arg \max \left(d V_{\mathrm{i}}(t) / d t\right)$ [17]. In the ECG, T-peak $t_{\mathrm{i}}^{\mathrm{P}}(k)$ was defined as the apex of the T-wave, and T-end $t_{\mathrm{i}}^{\mathrm{E}}(k)$ as the instant for which the tangent of the T-wave at the point of maximum downslope crosses the baseline (Fig. 3). T-peak and T-peak-end intervals were defined as $I_{\mathrm{i}}^{\mathrm{P}}=$ $t_{\mathrm{i}}^{\mathrm{P}}(k)-t_{\mathrm{i}}^{0}(k)$ and $I_{\mathrm{i}}^{\mathrm{E}}=t_{\mathrm{i}}^{\mathrm{E}}(k)-t_{\mathrm{i}}^{\mathrm{P}}(k)$, while local repolarization intervals were $I_{\mathrm{i}}^{\mathrm{R}}=t_{\mathrm{i}}^{\mathrm{R}}(k)-t_{\mathrm{i}}^{0}(k)$. In the previous expressions, $t_{\mathrm{i}}^{0}(k)$ are the time at which the pacing stimuli were delivered (Fig. 1 and Fig. 3). Two metrics 

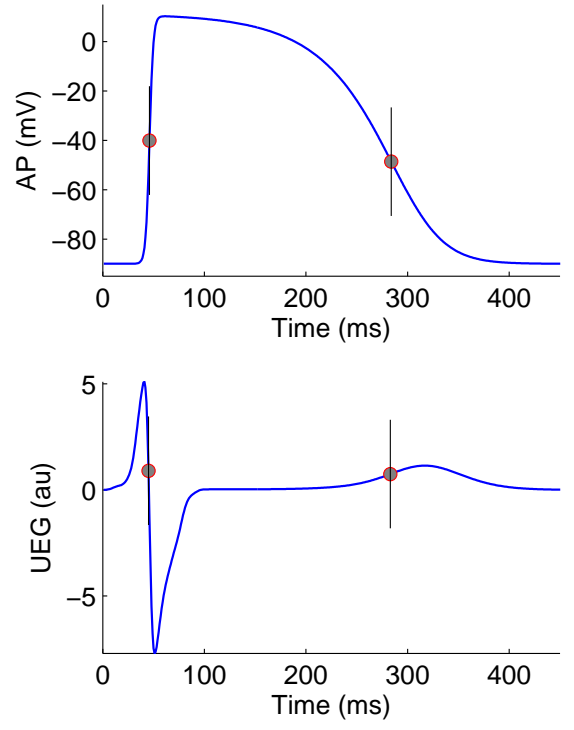

Figure 2. Measure of repolarization time of the action potential at maximum upslope in the local unipolar electrogram (diagrammatic).

were used to calculate reference values for SHVR from the UEGs as:

$\Delta \Sigma_{1}^{\mathrm{R}}=\operatorname{med}_{\mathrm{k}}\left[\mathrm{p}_{\mathrm{i}}^{80}\left[I_{\mathrm{i}}^{\mathrm{R}}(k)\right]\right] ; \quad \Delta \Sigma_{2}^{\mathrm{R}}=\operatorname{std}_{\mathrm{i}}\left[\operatorname{mean}_{\mathrm{k}}\left[I_{\mathrm{i}}^{\mathrm{R}}(k)\right]\right]$

while ECG-derived indices of SHVR were estimated as:

$$
\begin{aligned}
\Delta \Sigma_{1}^{\mathrm{P}} & =\operatorname{med}_{\mathrm{k}}\left[\mathrm{p}_{\mathrm{i}}^{80}\left[I_{\mathrm{i}}^{\mathrm{P}}(k)\right]\right] \\
\Delta \Sigma_{1}^{\mathrm{E}} & =\operatorname{med}_{\mathrm{k}}\left[\mathrm{p}_{\mathrm{i}}^{80}\left[I_{\mathrm{i}}^{\mathrm{E}}(k)\right]\right]
\end{aligned}
$$

In (4)-(6), $\operatorname{med}_{\mathrm{k}}$ and $\mathrm{p}_{\mathrm{i}}^{80}$ represent the median across heart beats and the interval from 10th to 90th percentile calculated across leads, respectively.

\section{4. $\quad$ Results}

Reference measures of SHVR from endo- and epicardial UEG were $\Delta \Sigma_{1}^{\mathrm{R}}=110.8 \pm 25.7 \mathrm{~ms}$ (mean $\pm \mathrm{std}$ ), $66.4-105.9-157.5 \mathrm{~ms}$ (min-median-max) and $\Delta \Sigma_{2}^{\mathrm{R}}=$ $44.9 \pm 9.85 \mathrm{~ms}$ (mean $\pm \mathrm{std}$ ), $30.4-45.5-62.0 \mathrm{~ms}$ (minmedian-max).

Figure 4 shows correlations between ECG-derived measures of $\operatorname{SHVR}\left(\mathcal{V}, \Delta \Sigma^{\mathrm{P}}\right.$ and $\left.\Delta \Sigma^{\mathrm{E}}\right)$ and reference measures $\Delta \Sigma^{\mathrm{R}}$. Pearson's $\left(r_{p}\right)$ and Spearman's $\left(r_{s}\right)$ correlation coefficients were in between 0.71 and 0.80 for $\mathcal{V}$ $\left(P<5 \cdot 10^{-4}\right), 0.29$ and 0.40 for $\Delta \Sigma^{\mathrm{P}}(0.10<P<0.05)$, and 0.22 and 0.36 for $\Delta \Sigma^{\mathrm{E}}(P>0.10)$. In comparison, correlation coefficients within intracardiac measures $\Delta \Sigma_{1}^{\mathrm{R}}$ and $\Delta \Sigma_{2}^{\mathrm{R}}$, were equal to 0.94 .

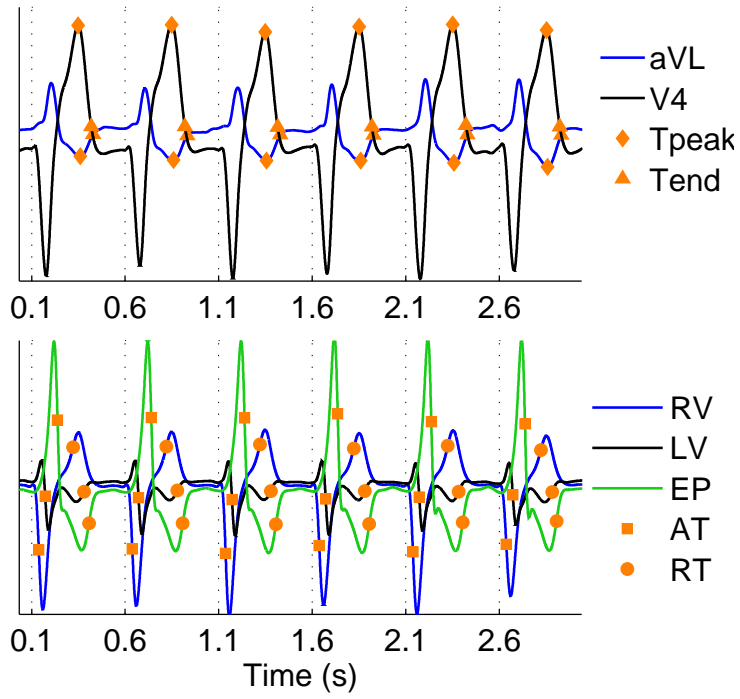

Figure 3. Illustrative example of data (band pass filtered) and markers of repolarization from the ECG, T-peak $t_{\mathrm{i}}^{\mathrm{P}}(k)$ and T-end $t_{\mathrm{i}}^{\mathrm{P}}(k)$ (top panel) and from the UEG, local repolarization time $t_{\mathrm{i}}^{\mathrm{R}}(k)$ (bottom panel).
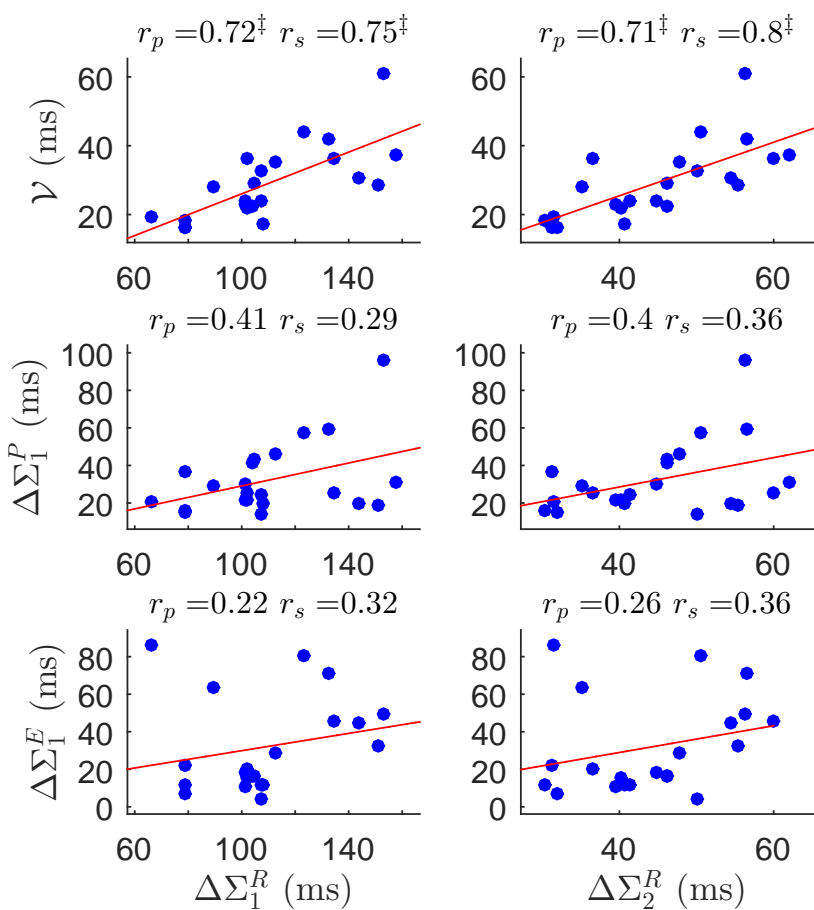

Figure 4. Correlation between ECG-derived measures of dispersion of repolarization $\mathcal{V}, \Delta \Sigma^{\mathrm{P}}$ and $\Delta \Sigma^{\mathrm{E}}$ and measures of dispersion of repolarization from intracardiac UEG, $\Delta \Sigma_{1}^{\mathrm{R}}$. Pearson's $\left(r_{p}\right)$ and Spearman's $\left(r_{s}\right)$ coefficients are also shown, with ( $\ddagger)$ significant correlation. Correlations for $\mathcal{V}$ were significant $\left(P<5 \cdot 10^{-4}\right)$, while for $\Delta \Sigma^{\mathrm{P}} 0.1<P<0.05$ and for $\Delta \Sigma^{\mathrm{E}} P>0.1 . \mathrm{N}=22$ recordings were analyzed. 


\section{Discussion and Conclusion}

Spatial heterogeneity of ventricular repolarization is an important factor influencing the arrhythmogenic properties of the ventricles. The V-index is an metric recently proposed to estimate SHVR non-invasively from the surface ECG [7]. A tight relation between V-index and the dispersion of repolarization has been demonstrated theoretically and using numerical simulations [9], and it has been confirmed in studies where patients were given drugs known to alter the dispersion of repolarization [8].

In this study, the V-index was compared with direct estimates of SHVR provided by unipolar elecrograms recorded in contact with the cardiac tissue, in the left and right ventricle and in the endo- and epicardium. The results of this study demonstrate that the V-index significantly correlates with direct measures of SHVR.

This study present some limitations. The relative small number of patients and recordings was mainly due to the invasiveness and cost of the procedure. Only recording during cardiac pacing were analyzed. This reduces the heart rate dependent temporal variability of repolarization, but it also alter the normal electrical conduction and recovery of the heart and it increases SHVR. Finally, although the reference values for SHVR included the dispersion of repolarization along the major axes (apex-base, right-left ventricles and endo-epicardium), direct measures of total SHVR ranging from the sites of earliest to the site of latest repolarization throughout the entire cardiac muscle may have been slightly different.

Altogether, this study adds further evidence regarding the reliability of the V-index as a non-invasive estimator of SHVR.

\section{References}

[1] Coronel R, Wilms-Schopman FJG, Opthof T, Janse MJ. Dispersion of repolarization and arrhythmogenesis. Heart Rhythm Apr 2009;6(4):537-543.

[2] Qu Z, Weiss J. Mechanisms of ventricular arrhythmias: From molecular fluctuations to electrical turbulence. Annual Review of Physiology 2015;77:29-55.

[3] Pueyo E, Martnez JP, Laguna P. Cardiac repolarization analysis using the surface electrocardiogram. Philos Transact A Math Phys Eng Sci Jan 2009;367(1887):213-233.

[4] Franz M, Zabel M. Electrophysiological basis of QT dispersion measurements. Prog Cardiovasc Dis 2000;42(5):311324.

[5] Meijborg VM, Conrath CE, Opthof T, Belterman CN, de Bakker JM, Coronel R. Electrocardiographic T wave and its relation with ventricular repolarization along major anatomical axes. Circulation Arrhythmia and Electrophysiology 2014;7(3):524-531.

[6] Porthan K, Viitasalo M, Toivonen L, Havulinna A, Jula A, Tikkanen J, Vnnen H, Nieminen M, Huikuri H, Newton-
Cheh C, Salomaa V, Oikarinen L. Predictive value of electrocardiographic $\mathrm{T}$-wave morphology parameters and $\mathrm{T}$ wave peak to T-wave end interval for sudden cardiac death in the general population. Circulation Arrhythmia and Electrophysiology 2013;6(4):690-696.

[7] Sassi R, Mainardi LT. An estimate of the dispersion of repolarization times based on a biophysical model of the ECG. IEEE Trans Biomed Eng Dec 2011;58(12):3396-3405.

[8] Rivolta M, Mainardi L, Sassi R. Quantification of ventricular repolarization heterogeneity during moxifloxacin or sotalol administration using V-index. Physiological Measurement 2015;36(4):803-811.

[9] Sassi R, Mainardi L, Laguna P, Rodriguez J. Validation of the Vindex through finite element 2d simulations 2013; 337-340.

[10] Sassi R, Rivolta MW, Mainardi LT, Reis RC, Rocha MOC, Ribeiro ALP, Lombardi F. Spatial repolarization heterogeneity and survival in chagas disease. Methods Inf Med 2014;53(6):464-468.

[11] Van Oosterom A. Genesis of the t wave as based on an equivalent surface source model. J Electrocardiol 2001; 34(SUPPL.):217-227.

[12] Finlay M, Xu L, Taggart P, Hanson B, Lambiase P. Bridging the gap between computation and clinical biology: Validation of cable theory in humans. Frontiers in Physiology 2013;4 SEP:-.

[13] Geselowitz DB. On the theory of the electrocardiogram 1989;77(6):857-876.

[14] Orini M, Citi L, Hanson BM, Taggart P, Lambiase PD. Characterization of the causal interactions between depolarization and repolarization temporal changes in unipolar electrograms 2013;719-722.

[15] Orini M, Hanson B, Monasterio V, Martinez J, Hayward M, Taggart P, Lambiase P. Comparative evaluation of methodologies for $\mathrm{T}$-wave alternans mapping in electrograms. Biomedical Engineering IEEE Transactions on 2014;61(2):308-316.

[16] Taggart P, Orini M, Hanson B, Hayward M, Clayton R, Dobrzynski H, Yanni J, Boyett M, Lambiase PD. Developing a novel comprehensive framework for the investigation of cellular and whole heart electrophysiology in the in situ human heart: Historical perspectives, current progress and future prospects. Prog Biophys Mol Biol Jun 2014;

[17] Western D, Hanson B, Taggart P. Measurement bias in activation-recovery intervals from unipolar electrograms. American Journal of Physiology Heart and Circulatory Physiology February 2015;308(4):H331-H338.

Address for correspondence:

M. Orini

Institute of Cardiovscular Science / University College London 132 Hampstead Road, London, United Kingdom

m.orini@ucl.ac.uk 